\title{
The safety of endoscopic esophageal procedures under general anesthesia
}

\author{
Go Eun Kim, Duk Kyung Kim, Ji Won Choi, In Sun Chung, and \\ Da Woon Jung \\ Department of Anesthesiology and Pain Medicine, Samsung Medical Center, Sungkyunkwan University School of \\ Medicine, Seoul, Korea
}

\begin{abstract}
Background: With the increasing demand for general anesthesia for endoscopic esophageal procedures, anesthesiologists should understand the clinical characteristics of post-procedural complications (PPCs).

Methods: We retrospectively investigated the incidence of and risk factors associated with PPCs of endoscopic esophageal procedures performed under general anesthesia from July 2013 to November 2016. The final analysis included 129 patients; 114 who underwent esophageal endoscopic dissection for esophageal tumors and 15 cases of peroral endoscopic myotomy for achalasia. Frank perforation during the procedure was defined as an endoscopically recognizable or clinically detected perforation during the procedures. A multivariable logistic regression analysis was conducted to identify independent risk factors for PPCs.

Results: The overall incidence of PPCs was 19.4\% (25/129). All of the PPCs were managed successfully with conservative measures. The most common PPC was symptomatic, radiologically documented atelectasis $(11 / 25,44.0 \%)$, followed by esophageal perforation-related PPCs (symptomatic pneumomediastinum or pneumoperitoneum; 9/25, 36.0\%). In the multivariable analysis, frank perforation during the procedure was the only independent risk factor for PPCs (odds ratio, 8.470, 95\% CI, 2.051-34.974, $\mathrm{P}=0.003$ ). Although frank perforation during the procedure occurred in 13 patients, $38.5 \%$ (5/13) of them did not develop any clinical sequelae after their procedures. Compared with patients without PPCs, patients who developed PPCs took longer to their first oral intake and had prolonged hospital stays $(\mathrm{P}=0.047$ and 0.026 , respectively).

Conclusions: Iatrogenic perforation during endoscopic esophageal procedures under general anesthesia was the only independent risk factor for PPCs; therefore, proactive measures and close follow-up are necessary.
\end{abstract}

Key Words: Complications, Esophagus, Gastrointestinal endoscopy, General anesthesia.

Corresponding author: Duk Kyung Kim, M.D., Ph.D.

Department of Anesthesiology and Pain Medicine, Samsung Medical Center, Sungkyunkwan University School of Medicine, 81, Irwon-ro, Gangnam-gu, Seoul 06351, Korea

Tel: 82-2-3410-0343, Fax: 82-2-3410-6626

Email: dikei@hanmail.net

ORCID: https://orcid.org/0000-0002-6555-2100

Received: January 18, 2017.

Revised: March 13, 2017 (1st); April 1, 2017 (2nd).

Accepted: April 3, 2017.

Korean J Anesthesiol 2017 October 70(5): 555-560

https://doi.org/10.4097/kjae.2017.70.5.555

\section{Introduction}

Endoscopic esophageal procedures such as endoscopic submucosal dissection (ESD) and peroral endoscopic myotomy (POEM) are increasingly being performed to avoid the considerable morbidity and mortality associated with esophageal surgery. With its wide application as one of the main therapeutic modalities for early gastric cancer, ESD is now accepted as a useful, minimally invasive procedure for the management of early esophageal cancer [1]. Although POEM is a relatively new procedure, technological advances have led to its widespread use for

(c) This is an open-access article distributed under the terms of the Creative Commons Attribution Non-Commercial License (http://creativecommons.org/ licenses/by-nc/4.0/), which permits unrestricted non-commercial use, distribution, and reproduction in any medium, provided the original work is properly cited. 
the treatment of achalasia [2].

Compared with endoscopic gastric procedures, endoscopic esophageal procedures carry an increased risk of perforation or bleeding due to the thinner esophageal wall, and motion with respiration and heartbeat in a narrow space [3]. Ventilatory support during these procedures is also challenging, as access to the patient's airway is not always readily achieved. In addition, patients undergoing these procedures frequently have comorbidities, including cardiopulmonary disease, or pathology that increases the risk of regurgitation and aspiration [4]. Therefore, unlike endoscopic gastric procedures (mostly performed under sedation), intubation and general anesthesia may be preferable for endoscopic esophageal procedures.

Despite growing demand to provide anesthetic service for endoscopic esophageal procedures outside the operating room, few reports have focused on the safety of endoscopic esophageal procedures performed under general anesthesia. Therefore, we retrospectively investigated the incidence of and risk factors associated with post-procedural complications (PPCs) of endoscopic esophageal procedures performed under general anesthesia in a single South Korean institution.

\section{Materials and Methods}

With Institutional Review Board approval (IRB file number: 2017-01-034; approval date: January 6, 2017), we retrospectively examined the records of patients aged $\geq 18$ years who received general anesthesia for endoscopic esophageal procedures from July 2013 to November 2016 at our institution.

No esophageal procedure under general anesthesia was repeated in the same patient. Therefore, this retrospective cohort study enrolled 129 patients: 114 ESDs for esophageal tumors and 15 POEMs for achalasia. All procedures were performed in the endoscopy suite located outside the main operating theater.

\section{Anesthesia}

In all cases, anesthesia care was provided by a dedicated team of anesthesiologists with extensive experience in gastrointestinal endoscopy. The POEM patients took nothing by mouth except clear fluids for $24 \mathrm{~h}$ beforehand, while the ESD patients were fasted for $8 \mathrm{~h}$ before their procedures.

Standard anesthesia induction and intubation were performed in all patients. After an intravenous bolus of 2-3 $\mathrm{mg}$ of midazolam, anesthesia was induced and maintained by continuous infusion of propofol and remifentanil using a targetcontrolled infusion pump or continuous infusion of dexmedetomidine and remifentanil. Rocuronium was administered at $0.6-0.9 \mathrm{mg} / \mathrm{kg}$ as a muscle relaxant. After tracheal intubation, the lungs were ventilated at a tidal volume of $8 \mathrm{ml} / \mathrm{kg}$ in volume- controlled mode. The respiratory rate was set to maintain the end-tidal carbon dioxide $\left(\mathrm{ETCO}_{2}\right)$ at $35-40 \mathrm{mmHg}$.

After completing the procedures, any residual neuromuscular block was reversed and the tracheal tube was removed in the endoscopy room. Then, the patients were transferred to the endoscopy recovery room.

In the recovery room, all patients underwent serial assessments of pain using an 11-point numerical rating scale (NRS). When the patient complained of a pain NRS score $\geq 5,25 \mathrm{mg}$ of meperidine was injected intravenously. Patients were discharged to the ward when they had a modified Aldrete score $\geq 9$.

\section{Endoscopic procedures}

Before every procedure, $5 \mathrm{mg}$ of cimetropium was administered intravenously. All endoscopic procedures were performed with a video endoscope system (EVIS LUCERA ELITE; Olympus, Tokyo, Japan) by one of three experienced endoscopists. During the procedures, $\mathrm{CO}_{2}$ insufflation was maintained at a rate of $1.2-1.5 \mathrm{~L} / \mathrm{min}$.

As described previously [5], the ESD procedure sequence typically consisted of marking the mucosa, making a mucosal incision, and performing submucosal dissection with simultaneous hemostasis. The POEM procedure sequence consisted of mucosal entry after the injection of normal saline mixed with epinephrine and indigo carmine, submucosal tunneling, and a myotomy using a Triangle Tip Knife (KD-640 L; Olympus, Tokyo, Japan), and hemostatic clip application to close the mucosal entry site.

For both procedures, all patients underwent chest radiography on the day of the procedure and the first day after the procedure to screen for pneumonia and perforation.

\section{Variable selection and definition}

PPCs included any of the following: pulmonary complications (atelectasis, effusion, aspiration or infectious pneumonia, pulmonary embolism, and acute respiratory failure), cardiovascular complications (critical arrhythmias, angina pectoris, myocardial infarction, congestive heart failure, and thromboembolic events), bleeding that required transfusion or emergency endoscopic/surgical intervention, and complications induced by procedure-related perforation (pneumothorax and symptomatic pneumomediastinum or pneumoperitoneum). Pulmonary complications were diagnosed only if supported by both clinical and radiological evidence.

The following clinical characteristics were collected using medical records: patient-related (demographic variables, American Society of Anesthesiologists physical status, history of smoking, and alcohol use), lesion-related (location of lesion), 
Table 1. Univariable Analyses of the Clinical Characteristics of the Patients with and without Post-procedural Complications (PPCs)

\begin{tabular}{|c|c|c|c|}
\hline & $\begin{array}{l}\text { With PPCs } \\
(\mathrm{n}=25)\end{array}$ & $\begin{array}{l}\text { Without PPCs } \\
\quad(\mathrm{n}=104)\end{array}$ & $P$ value \\
\hline $\operatorname{Sex}(M / F)$ & $22 / 3$ & $97 / 7$ & 0.376 \\
\hline Age (yr) & $60.9 \pm 12.7$ & $63.8 \pm 10.6$ & 0.238 \\
\hline Current smoking/ex- or non-smoking & $4 / 21$ & $30 / 74$ & 0.191 \\
\hline Alcohol abuse (yes/no) & $6 / 19$ & $36 / 68$ & 0.309 \\
\hline $\operatorname{BMI}\left(\mathrm{kg} / \mathrm{m}^{2}\right)$ & $23.3 \pm 3.1$ & $24.0 \pm 2.4$ & 0.174 \\
\hline ASA physical status (I/II/III) & $8 / 14 / 3$ & $34 / 65 / 5$ & 0.402 \\
\hline $\begin{array}{l}\text { Location of lesion (cervical/upper thoracic/mid-thoracic/lower thoracic } \\
\text { and intra-abdominal) }\end{array}$ & $0 / 2 / 16 / 7$ & $0 / 12 / 52 / 40$ & 0.452 \\
\hline Procedure type (ESD/POEM) & $19 / 6$ & $95 / 9$ & $0.032 *$ \\
\hline Anesthesia type $(\mathrm{PPF}+\mathrm{Remi} / \mathrm{Dex}+\mathrm{Remi})$ & $20 / 5$ & $95 / 9$ & 0.101 \\
\hline Duration of surgery (min) & $86.0 \pm 37.8$ & $73.3 \pm 48.3$ & 0.164 \\
\hline Duration of anesthesia (min) & $113.5 \pm 39.5$ & $97.0 \pm 49.1$ & 0.081 \\
\hline Frank perforation during procedure (yes/no) & $8 / 17$ & $5 / 99$ & $<0.001^{*}$ \\
\hline Maximum pain NRS score in the recovery room $(0-10)$ & $0.0(0.0-7.0)$ & $1.0(0.0-6.0)$ & 0.793 \\
\hline Total dose of meperidine used in the recovery room $(\mathrm{mg})$ & $0.0(0.0-25.0)$ & $0.0(0.0-25.0)$ & 0.647 \\
\hline
\end{tabular}

All data are expressed as the mean \pm SD, median (interquartile range), or number. BMI: body mass index, ASA: American Society of Anesthesiologists, ESD: endoscopic submucosal dissection, POEM: peroral endoscopic myotomy, PPF: propofol, Remi: remifentanil, Dex: dexmedetomidine, NRS: numeric rating scale. ${ }^{*}$ Statistically significant difference $(\mathrm{P}<0.05)$.

anesthesia-related (anesthesia methods, duration of anesthesia, maximum pain NRS score in the recovery room, and total dose of meperidine used for post-procedural pain in the recovery room), and procedure-related (procedure type, duration of procedure, and frank perforation during the procedure).

Alcohol abuse was defined as an average of 3-4 drinks per day, four or more times per week, and current smoking as having smoked at least one cigarette per day for more than 1 year within at least 6 weeks before surgery. Frank perforation during the procedure was defined as endoscopically recognizable perforation or perforation diagnosed by severe subcutaneous emphysema, pneumomediastinum, or pneumoperitoneum during the procedures. The post-procedural recovery profile (length of recovery room stay, time to first oral intake, and length of hospital stay) was also documented.

\section{Statistical analysis}

First, univariable analyses were performed to assess the associations of each clinical characteristic with PPCs. Continuous variables were tested for normality using the KolmogorovSmirnov test. Non-normally and normally distributed continuous variables were analyzed with the Mann-Whitney $U$ test and unpaired $t$-test, respectively. Categorical variables were analyzed with the $\chi^{2}$ or Fisher's exact test, as appropriate.

Then, forward stepwise multivariable logistic regression analysis was used to identify independent predictors of PPCs. Variables that were significant at $\mathrm{P} \leq 0.2$ in the univariable analyses were considered to enter in the multivariable analysis. Cox and Snell's $\mathrm{R}^{2}$ and Nagelkerke's $\mathrm{R}^{2}$ were computed as measures of the
Table 2. Classification of Post-procedural Complications of Endoscopic Esophageal Procedures

\begin{tabular}{lc}
\hline \multicolumn{1}{c}{ Classification } & Number of patients \\
\hline Pulmonary complications & 11 \\
Atelectasis & 3 \\
Aspiration pneumonia & 1 \\
$\quad$ Viral pneumonia & 1 \\
Cardiovascular complications & \\
Perforation-related complications & 4 \\
Symptomatic pneumomediastinum only & 3 \\
Symptomatic pneumoperitoneum only & 2 \\
Symptomatic pneumomediastinum & \\
$\quad$ combined with pneumoperitoneum & \\
\hline
\end{tabular}

goodness-of-fit of the regression model. The odds ratios, 95\% $\mathrm{CI}$, and $\mathrm{P}$ value of the independent predictors were calculated. SPSS Statistics ver. 20 (IBM Corp., Armonk, NY, USA) was used for all analyses. Statistical significance was set at $\mathrm{P}<0.05$.

\section{Results}

This study enrolled 129 patients; 119 men and 10 women (Table 1). The median (interquartile range) age of all patients was 66.0 (57.0-71.0) years.

The overall incidence of PPCs was 19.4\% (25/129). The most common PPC was symptomatic, radiologically documented atelectasis $(\mathrm{n}=11)$. Additional pulmonary complications included aspiration $(n=3)$ and viral $(n=1)$ pneumonia (Table 2$)$. All patients who developed aspiration pneumonia underwent ESD.

Nine patients developed symptomatic pneumomediastinum 
or pneumoperitoneum on the day of the procedure, which was resolved by conservative management (fasting and intravenous antibiotics). Of these, one patient did not show any signs of $\mathrm{CO}_{2}$ leakage from the esophagus during the procedure. Two of these patients had simultaneous pneumomediastinum and pneumoperitoneum. One cardiovascular complication occurred, namely, cardiogenic shock necessitating cardioversion that developed in a patient with pre-existing atrial fibrillation.

No reoperation due to procedural complications or mortality occurred during hospitalization. There were no general anesthesia-specific adverse events, such as adverse drug reactions or intubation complications.

Frank perforation during the procedure occurred in 13 patients, of which six patients were identified only when they developed severe subcutaneous emphysema or pneumoperitoneum (without endoscopic evidence of perforation). Except for one case requiring polyglycolic acid sheet application, all endoscopically recognized perforations were treated endoscopically by applying endoclips. Overall, 38.5\% (5/13) of the patients with a frank perforation did not develop any clinical sequelae on the ward (Table 3).

\section{Univariable and multivariable analysis}

Of the variables studied, procedure type (POEM vs. ESD) and frank perforation during the procedure were significantly associated with PPCs in the univariable analyses $(\mathrm{P}<0.05$, Table 1$)$.

Given the high correlation between 'duration of procedure' and 'duration of anesthesia' (Pearson's correlation coefficient = $0.987, \mathrm{P}<0.001$ ), the latter variable was removed. Thus, in addition to these two variables, four variables associated with PPCs
$(\mathrm{P} \leq 0.2)$ in the univariate analysis (current smoking, body mass index, anesthesia type, and procedure duration) were entered into the multivariate logistic regression analysis. Of these, only frank perforation during the procedure was an independent risk factor for PPCs (odds ratio, 8.470, 95\% CI, 2.051-34.974, P = $0.003)$.

\section{Recovery variables between patients with and without PPCs}

Post-procedures, the patients who developed PPCs had a longer time to first oral intake $(2.5 \pm 2.8$ and $1.3 \pm 0.8$ days for patients with and without PPCs, respectively; $\mathrm{P}=0.047)$. In addition, the presence of a PPC resulted in a prolonged postprocedural hospital stay $(4.9 \pm 3.5$ and $3.2 \pm 0.7$ days for patients with and without PPCs, respectively; $\mathrm{P}=0.026$ ) (Table 4).

\section{Discussion}

The main finding of this study was that PPCs occurred with an incidence of $19.4 \%$ in patients undergoing endoscopic esophageal procedures with general anesthesia, but all of these complications could be dealt with during the procedures and managed successfully by conservative measures after the procedures.

In the era of endoscopic gastric procedures, the safety and efficacy of sedation are clear [6,7]. While one study reported a favorable result for sedation [8], advanced esophageal endoscopy is generally performed under general anesthesia. Compared with endoscopic gastric procedures, minimal patient movement is more important during endoscopic esophageal procedures due to the anatomic differences [3]. Insufficient depth of seda-

Table 3. Characteristics and Clinical Courses of the Patients with Frank Perforation during the Procedure

\begin{tabular}{lc}
\hline & Number of patients \\
\hline Endoscopically recognizable perforation (yes/no) & $7 / 6$ \\
ESD/POEM & $8 / 5$ \\
No treatment/closure with clips/application of polyglycolic acid sheets & $6 / 6 / 1$ \\
Application of abdominal drain (yes/no) & $1 / 12$ \\
Progression to PPCs (yes/no) & $8 / 5$ \\
\hline
\end{tabular}

ESD: endoscopic submucosal dissection, POEM: peroral endoscopic myotomy, PPCs: post-procedural complications.

Table 4. Recovery Variables of the Patients with and without Post-procedural Complications (PPCs)

\begin{tabular}{lccc}
\hline & $\begin{array}{c}\text { With PPCs } \\
(\mathrm{n}=25)\end{array}$ & $\begin{array}{c}\text { Without PPCs } \\
(\mathrm{n}=104)\end{array}$ & P value \\
\hline Recovery room stay (min) & $34.8 \pm 9.8$ & $34.1 \pm 8.0$ & 0.703 \\
Time to first oral intake (days) & $2.5 \pm 2.8$ & $1.3 \pm 0.8$ & $0.047^{*}$ \\
Postoperative hospital stay (days) & $4.9 \pm 3.5$ & $3.2 \pm 0.7$ & $0.026^{*}$ \\
\hline
\end{tabular}

All data are expressed as mean $\pm \mathrm{SD}$. *Statistically significant difference $(\mathrm{P}<0.05)$. 
tion can lead to an unstable respiratory status or patient movement, which may result in complications such as perforation and hemorrhage.

In addition to the absence of patient movement, the major advantages of general anesthesia for endoscopic esophageal procedures include the reliability of the airway with tracheal intubation, a reduced risk of aspiration pneumonia, and the assistance of an anesthesiologist if complications occur [3,9]. As a result, more anesthesiologists are likely to encounter such patients in their daily practice.

Compared with gastric ESD, the risk of perforation is greater for esophageal ESD, with perforation rates in gastric ESD ranging between $1.2 \%$ and $5.2 \%$ versus up to $10.7 \%$ in esophageal ESD [10,11]. Although one small retrospective study [3] reported a similar incidence of perforation between general anesthesia and sedation, it is uncertain whether anesthesia methods influence the risk of perforation during esophageal ESD. In comparison, the reported perforation rate in POEM ranges broadly from $0.4 \%$ to $14 \%$ [11].

Although the majority of esophageal perforations can be treated successfully with immediate endoscopic closure and conservative management, it is considered a major complication due to the potential for severe emphysema and life-threatening mediastinal inflammation. In this study, perforation-related PPCs occurred in nine cases (7.0\% of all cases). Fortunately, all of them resolved with endoscopic closure and conservative measures.

Notably, 38.5\% (5/13) of the patients with a frank perforation did not show any symptoms or signs on the ward. This result may be in part attributed to the use of $\mathrm{CO}_{2}$ gas during the endoscopic esophageal procedures. $\mathrm{CO}_{2}$ gas is cleared from the gastrointestinal tract more rapidly than air and is subsequently exhaled from the lungs [12]. $\mathrm{CO}_{2}$ insufflation during endoscopic esophageal procedures is known to reduce the risk of severe perforation-related PPCs [12]. In addition, general anesthesia itself may exert a protective effect on the progression of pneumoperitoneum or pneumomediastinum. The mediastinal pressure is higher than the intra-esophageal pressure under general anesthesia, which prevents mediastinal emphysema.

In this study, frank perforation during the procedure was found to be a strong independent risk factor for PPCs. As demonstrated in this study, the presence of frank perforation during the procedure does not always precede clinically significant PPCs. However, $61.5 \%$ (8/13) of the cases with a frank perforation progressed to symptomatic pneumomediastinum or pneumoperitoneum. It is evident that the clinical outcome of iatrogenic perforation depends on the timing and efficacy of interventions. In the case of an endoscopically identified or clinically suspected perforation, proactive intervention, including immediate treatment with endoclips, is necessary to minimize the morbidity and mortality related to iatrogenic perforation $[11,13]$. Careful follow-up is mandatory in cases with a prolonged high-grade fever, positive tests for inflammation, and chest pain.

Post-procedural pneumomediastinum or pneumoperitoneum may occur in the absence of endoscopically or clinically detected perforation during ESD or POEM. In our series, one patient who developed symptomatic pneumomediastinum did not show any sign of $\mathrm{CO}_{2}$ leakage from the esophagus during the procedure. This may be attributed to the absence of serosa in the esophagus. $\mathrm{CO}_{2}$ gas might diffuse into the mediastinum or abdomen via muscle fibers exposed during the submucosal dissection or myotomy $[13,14]$. However, this is usually self-limiting because $\mathrm{CO}_{2}$ gas is reabsorbed spontaneously after the procedure. If clinically relevant on the ward, such a small amount of gas leakage can be effectively treated by using antibiotics and fasting.

In this study, three cases of aspiration pneumonia were observed. In addition to achalasia or an esophageal tumor, upper gastrointestinal endoscopy itself is a known risk factor for regurgitation and aspiration $[4,14,15]$. Aspiration pneumonia occurs in 1.6-6.6\% of gastric ESD cases under sedation [16,17]. Although not perfect, positive pressure ventilation with tracheal intubation significantly decreases the risk of regurgitation or aspiration. This is the main reason for the relatively low incidence of aspiration pneumonia observed in this study.

Some anesthesiologists recommended the routine preanesthetic clearance of the esophageal contents before POEM because the prevention of aspiration is more important during POEM [15]. Nevertheless, no aspiration pneumonia developed in the POEM patients in this study, although prior endoscopic clearance was not performed. We believe that withholding oral intake except clear fluids for $24 \mathrm{~h}$ before POEM might have protective effects on regurgitation and aspiration. Routine preanesthetic endoscopic suctioning may cause marked discomfort in some patients.

In our series, there were no general anesthesia-specific adverse effects such as adverse drug reactions or intubation complications. Atelectasis, the most common PPC in this study, can occur following non-surgical procedures under sedation. In a retrospective analysis of upper gastrointestinal endoscopic resection (mostly including gastric lesions), an atelectasis rate of $19.5 \%$ (68 of 349 cases) was reported after endoscopist-directed sedation [18]. Compared with that study, we had a relatively low incidence of atelectasis $(11 / 129,8.5 \%)$, although direct comparison may be impossible due to the different procedures studied. Further study is necessary to clarify the beneficial effect of general anesthesia over sedation on atelectasis following endoscopic esophageal procedures.

One major limitation of this study is that it was a retrospec- 
tive observational study performed in a single institution. In South Korea, general anesthesia is administered for endoscopic esophageal procedures only in a few hospitals because of a shortage of anesthesiologists. Therefore, our study should help the anesthesia community to cope actively with the increasing demand for remote anesthesia services for endoscopic esophageal procedures. Another limitation is the relatively small number of POEM patients. As POEM is a new endoscopic intervention, experience with it is very limited globally.

In conclusion, this study demonstrated that procedurespecific complications, such as esophageal perforation-related PPCs (pneumomediastinum or pneumoperitoneum) and aspiration pneumonia, were not uncommon following endoscopic esophageal procedures performed under general anesthesia. Many complications could be controlled safely during the procedures and managed conservatively afterwards. However, as frank perforation during the procedure was found to be a strong independent risk factor for PPCs, proactive interventions and close follow-up are necessary to minimize the morbidity and mortality related to iatrogenic perforation in such cases.

\section{ORCID}

Duk Kyung Kim, https://orcid.org/0000-0002-6555-2100

Ji Won Choi, https://orcid.org/0000-0001-7403-2863

In Sun Chung, https://orcid.org/0000-0002-2874-0225

\section{References}

1. Kim JS, Kim BW, Shin IS. Efficacy and safety of endoscopic submucosal dissection for superficial squamous esophageal neoplasia: a metaanalysis. Dig Dis Sci 2014; 59: 1862-9.

2. Zhang XC, Li QL, Xu MD, Chen SY, Zhong YS, Zhang YQ, et al. Major perioperative adverse events of peroral endoscopic myotomy: a systematic 5-year analysis. Endoscopy 2016; 48: 967-78.

3. Yagi Kuwata N, Gotoda T, Suzuki S, Mukai S, Itoi T, Moriyasu F. Reasonable decision of anesthesia methods in patients who underwent endoscopic submucosal dissection for superficial esophageal carcinoma: A retrospective analysis in a single Japanese institution. Turk J Gastroenterol 2016; 27: 91-6.

4. Yang M, Ahn HJ, Kim JA, Yu JM. Risk score for postoperative complications in thoracic surgery. Korean J Anesthesiol 2012; 63: 527-32.

5. Lee H, Yun WK, Min BH, Lee JH, Rhee PL, Kim KM, et al. A feasibility study on the expanded indication for endoscopic submucosal dissection of early gastric cancer. Surg Endosc 2011; 25: 1985-93.

6. Sasaki T, Tanabe S, Ishido K, Azuma M, Katada C, Higuchi K, et al. Recommended sedation and intraprocedural monitoring for gastric endoscopic submucosal dissection. Dig Endosc 2013; 25 Suppl 1: 79-85.

7. Yoo YC, Park CH, Shin S, Park Y, Lee SK, Min KT. A comparison of sedation protocols for gastric endoscopic submucosal dissection: moderate sedation with analgesic supplementation vs analgesia targeted light sedation. Br J Anaesth 2015; 115: 84-8.

8. Nonaka T, Inamori M, Miyashita T, Harada S, Inoh Y, Kanoshima K, et al. Feasibility of deep sedation with a combination of propofol and dexmedetomidine hydrochloride for esophageal endoscopic submucosal dissection. Dig Endosc 2016; 28: 145-51.

9. Yamashita K, Shiwaku H, Ohmiya T, Shimaoka H, Okada H, Nakashima R, et al. Efficacy and safety of endoscopic submucosal dissection under general anesthesia. World J Gastrointest Endosc 2016; 8: 466-71.

10. Libânio D, Pimentel-Nunes P, Dinis-Ribeiro M. Complications of endoscopic resection techniques for upper GI tract lesions. Best Pract Res Clin Gastroenterol 2016; 30: 735-48.

11. Paspatis GA, Dumonceau JM, Barthet M, Meisner S, Repici A, Saunders BP, et al. Diagnosis and management of iatrogenic endoscopic perforations: European Society of Gastrointestinal Endoscopy (ESGE) Position Statement. Endoscopy 2014; 46: 693-711.

12. Maeda Y, Hirasawa D, Fujita N, Ohira T, Harada Y, Yamagata T, et al. Carbon dioxide insufflation in esophageal endoscopic submucosal dissection reduces mediastinal emphysema: A randomized, double-blind, controlled trial. World J Gastroenterol 2016; 22: 7373-82.

13. Tamiya Y, Nakahara K, Kominato K, Serikawa O, Watanabe Y, Tateishi H, et al. Pneumomediastinum is a frequent but minor complication during esophageal endoscopic submucosal dissection. Endoscopy 2010; 42: 8-14.

14. Yang S, Zeng MS, Zhang ZY, Zhang HL, Liang L, Zhang XW. Pneumomediastinum and pneumoperitoneum on computed tomography after peroral endoscopic myotomy (POEM): postoperative changes or complications? Acta Radiol 2015; 56: 1216-21.

15. Tanaka E, Murata H, Minami H, Sumikawa K. Anesthetic management of peroral endoscopic myotomy for esophageal achalasia: a retrospective case series. J Anesth 2014; 28: 456-9.

16. Akasaka T, Nishida T, Tsutsui S, Michida T, Yamada T, Ogiyama H, et al. Short-term outcomes of endoscopic submucosal dissection (ESD) for early gastric neoplasm: multicenter survey by osaka university ESD study group. Dig Endosc 2011; 23: 73-7.

17. Watari J, Tomita T, Toyoshima F, Sakurai J, Kondo T, Asano H, et al. The incidence of "silent" free air and aspiration pneumonia detected by CT after gastric endoscopic submucosal dissection. Gastrointest Endosc 2012; 76: 1116-23.

18. Choe JW, Jung SW, Song JK, Shim E, Choo JY, Kim SY, et al. Predictive factors of atelectasis following endoscopic resection. Dig Dis Sci 2016; 61: 181-8. 